\title{
Sensitivity of large cosmic ray air shower experiments for new physics searches
}

\author{
M.C. Espirito Santo*, M. Paulos, M. Pimenta, J.C. Romão, B. Tomé ${ }^{\dagger}$ \\ LIP and IST, Av. Elias Garcia, 14-1, 1000-149 Lisboa Portugal \\ E-mail: \\ catarina@lip.pt, paulos@lip.pt, pimenta@lip.pt, jorge.romao@ist.utl.pt, \\ bernardodip.pt
}

\section{Cardoso}

CFC, Universidade de Coimbra, P-3004-516 Coimbra, Portugal

E-mail: vcardosoefisica.ist.utl.pt

\section{A. Onofre}

LIP and Universidade Católica, Figueira da Foz, Portugal

E-mail: antonio.onofre@cern.ch

\begin{abstract}
Searches for physics beyond the Standard Model of particle physics are performed at accelerators worldwide. Although having poorer detection capabilities and large beam uncertainties, ultra high energy cosmic ray (UHECR) experiments present a unique opportunity to look for new physics far beyond the TeV. Nearly horizontal energetic neutrinos, seeing a large atmospheric target volume and with negligible background from "ordinary" cosmic rays, are ideal to explore rare processes. The sensitivity of present and planned experiments to different new physics scenarios is estimated, including mini black-holes, excited leptons and leptoquarks.
\end{abstract}

International Europhysics Conference on High Energy Physics

July 21st - 27th 2005

Lisboa, Portugal

\footnotetext{
* Speaker.

${ }^{\dagger}$ FCT grant SFRH/BPD/11547/2002.

†FT grant SFRH/BPD/14483/2003.
} 


\section{Introduction}

Cosmic ray experiments present a unique opportunity to look for new physics at scales far beyond the TeV. Energetic cosmic particles interact with the atmosphere originating Extensive Air Showers (EAS). While particles with strong or electromagnetic charges are rapidly absorbed, neutrinos have a much lower interaction cross-section and can easily travel large distances. Energetic cosmic neutrinos, although not yet observed and with large uncertainties on the expected fluxes, are predicted on rather solid grounds [1]. Nearly horizontal neutrinos, seeing a large target volume and with negligible background from "ordinary" cosmic rays, are thus an ideal beam to explore possible rare processes [2]. With large extra dimensions in our universe, black holes (BH) could be produced in UHECR atmospheric interactions. Events with a double bang topology, where the production and decay of a microscopic BH (first bang) is followed at measurable distance by the decay of an energetic tau lepton (second bang) could be an almost background free signature. Compositeness is a never discarded hypothesis for explaining the complexity of the fundamental particle picture; leptoquarks arise naturally in models unifying the quark and lepton sectors. Such particles could be produced in interactions of quasi-horizontal cosmic neutrinos with the atmosphere, originating detectable EAS. The capabilities of current (AGASA [3], Fly's Eye [4]) and future (Auger [5], EUSO [6], OWL [7]) very high energy cosmic ray experiments to detect these phenomena are discussed.

\section{Microscopic black hole detection - the double bang signature}

In the proposed scenario energetic neutrinos $\left(E_{v} \sim 10^{6}-10^{12} \mathrm{GeV}\right)$ interact deeply in the atmosphere (cross-section $\sim 10^{3}-10^{7} \mathrm{pb}$ ) producing microscopic BH with a mass of the order of the neutrino-parton center-of-mass energy $(\sqrt{s} \sim 1-10 \mathrm{TeV})$. An instantaneous thermal and democratic decay can be assumed. The average decay multiplicity $(N)$ is a function of the parameters of the model (Planck mass $M_{D}$, BH mass $M_{B H}$, number of extra dimension $n$ ) and typical values of the order of 5-20 are obtained in large regions of the parameter space. A large fraction of the decay products are hadrons but there is a non negligible number of charged leptons $(\sim 10 \%)[8,9]$. The energy spectra of such leptons in the BH centre-of-mass reference frame peaks around $M_{B H} / N$. Tau leptons provide a "golden" signature for microscopic BH detection in horizontal air shower events [10]. In fact, in the relevant energy range, the tau interaction length in air is much higher than its decay length [8]. A detectable second bang can be produced for tau leptons with a decay length large enough for the two bangs to be well separated, but small enough for a reasonable percentage of decays to occur within the field of view. Another critical aspect for the detectability of the second bang is the visible energy in the tau decay, and only decays into hadrons or electrons originate extensive air showers, leading to observable signals. However, the energy threshold for this second shower is only determined by the expected number of signal and background photons in a very restricted region of the field of view, as the second shower must be aligned with the direction of the first one. Double bang events in EUSO were generated parameterising the shower development and the atmosphere response as detailed in [10]. The modified frequentist likelihood ratio method [11], which takes into account the shapes of the signal and background distributions, was used to compute the statistical significance of the second shower. An expected background 
rate of 300-500 photons $/\left(\mathrm{m}^{2}\right.$.ns.sr) was considered [12]. An ideal photon detection efficiency of 1.0 and a more realistic one of 0.1 were taken. The fraction of the $\mathrm{BH}$ events with a first bang within the EUSO field of view that also have an observable second shower is shown in fig. 1(a).

\section{Sensitivity for excited lepton and leptoquark detection}

Excited leptons could be produced in neutrino-parton collisions via neutral (NC) and charged current (CC) processes, $v N \rightarrow v^{*} X$ and $v N \rightarrow \ell^{*} X$. The hadronic component $X$, and possibly part of the excited lepton decay products, would originate an EAS. The strength of the coupling between excited leptons and the SM leptons is parameterised through the weight factors $f$ and $f^{\prime}$ and the compositeness scale parameter, $\Lambda$. The total CC and NC cross-sections were computed from the neutrino-parton cross-section, as detailed in [14]. For $E_{v}=10^{20} \mathrm{eV}$ and $f / \Lambda=15 \mathrm{TeV}^{-1}$ they range between $50 \mathrm{nb}-100 \mathrm{nb}$ (between $1 \mathrm{nb}-2 \mathrm{nb}$ ) for an excited lepton mass of $m_{*}=1 \mathrm{TeV} / c^{2}$ $\left(m_{*}=100 \mathrm{TeV} / c^{2}\right)$. Excited leptons are assumed to decay promptly by radiating a $\gamma, \mathrm{W}^{ \pm}$or $\mathrm{Z}^{0}$ boson. In cosmic ray air shower experiments, only the excited lepton decay products originating hadronic or electromagnetic showers will contribute to the EAS. High energy taus may produce double bang signatures of the type described above.

Different leptoquark types are expected, according to their quantum numbers [15], which give rise to different coupling strengths and decay modes, and thus to different cross-sections and final states. If the available energies are high enough, the interaction of cosmic neutrinos with the atmospheric nuclei should create the ideal conditions for the production of leptoquarks, with dominance of $s$-channel resonant production. The produced leptoquarks are expected to decay promptly into a quark and a charged or neutral lepton. The branching ratio into the charged and neutral decay mode depends on the leptoquark type.

The expected number of observed events was obtained from the computed cross-sections, assuming the Waxman-Bahcall (WB) [16] bound with no $\mathrm{z}$ evolution for the incident neutrino flux, $E_{v}^{2} \frac{d \phi}{d E_{v}}=10^{-8}\left[\mathrm{GeV} / \mathrm{cm}^{2} \mathrm{~s} \mathrm{sr}\right]$. The procedure outlined in [14] was followed to obtain estimations of the acceptances of the different experiments. The relation between the shower energy and the primary neutrino energy is process dependent. For each scenario an average acceptance as a function of the incident neutrino energy, was computed via Monte Carlo taking into account the $d \sigma_{v N} / d y$ distributions and the different possible decay modes [14]. Fig. 1(b) shows the obtained sensitivities for excited electrons, as a function of the excited lepton mass.

\section{References}

[1] F. Halzen and D. Hooper, Rept. Prog. Phys. 65 (2002) 1025 [astro-ph/0204527]; O.E. Kalashev, V.A. Kuzmin, D.V. Semikoz, G. Sigl, Phys. Rev. D66 (2002) [hep-ph/0205050].

[2] C. Tyler, A.V. Olinto, G. Sigl Phys. Rev. D63 (2001) [hep-ph/0002257]; A. Ringwald, Invited talk at CRIS2004, Catania, May 2004 (2004) [hep-ph/0409151.]

[3] M. Takeda et al., Phys. Rev. Lett. 81, 1163 (1998), astro-ph/9807193;

N. Hayashida et al., astro-ph/0008102; http://www.akeno.icrr.u-tokyo.ac.jp/AGASA.

[4] D. J. Bird et al., Phys. Rev. Lett. 71 (1993) 3401; Astrophysics J. 424, 491 (1994); ibid. 441, 144 (1995) [astro-ph/9410067]. 

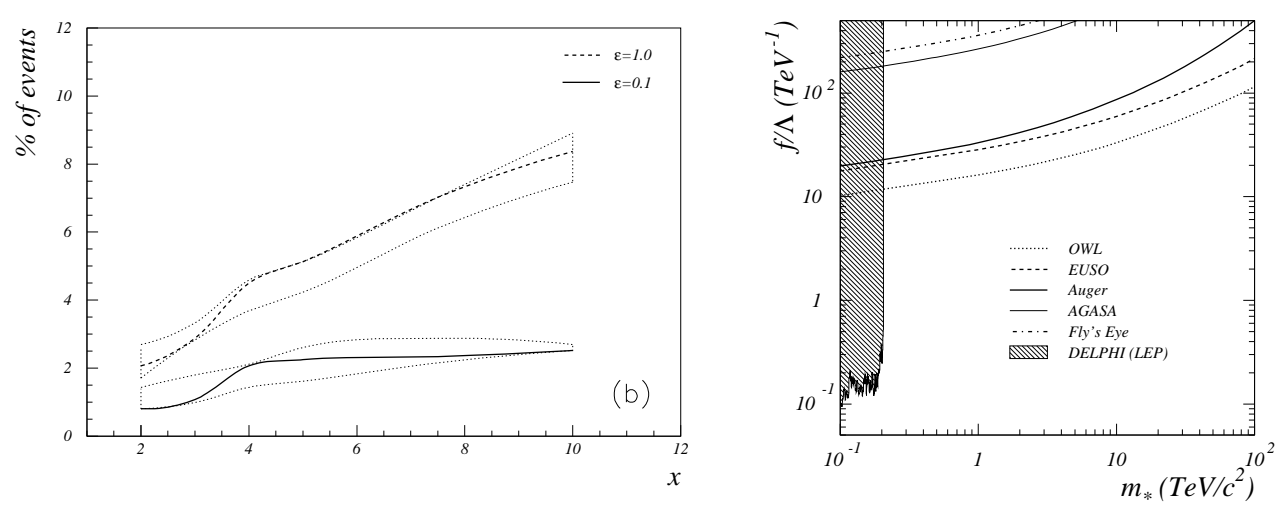

Figure 1: a) Fraction of the BH events with a first bang within the EUSO field of view that also have a visible second bang, as a function of $x=M_{B H} / M_{D}$, for $\mathrm{E}=10^{20} \mathrm{eV}$ and detection efficiencies $\varepsilon=0.1$ and $\varepsilon=1$. The thick lines correspond to $M_{D}=1 \mathrm{TeV}, n=3$ and the dotted bands give the variation of the results when varying $M_{D}$ between $1 \mathrm{TeV}$ and $2 \mathrm{TeV}$ and $n$ between 3 and $6 . \mathrm{b}$ ) Estimated sensitivities of the different experiments as a function of the excited lepton mass, for excited electrons in the $f=f^{\prime}$ scenario. The regions excluded by the DELPHI experiment at LEP are also shown (in dashed) for comparison [17].

[5] Auger Collab., The Pierre Auger Project Design Report, FERMILAB-PUB-96-024, 252 (1996). http://www.auger.org.

[6] O. Catalano, In Nuovo Cimento, 24-C, 2, 445 (2001); http://www.euso-mission.org; L. Scarsi et al., EUSO Collab., Report on the EUSO Phase A study, EUSO report EUSO-PI-REP-002-1 (2003).

[7] J. F. Krizmanic et al., OWL/AirWatch Collab., Proc. of the $26^{\text {th }}$ ICRC, Vol. 2, 388 (1999); http://owl.gsfc.nasa.gov

[8] J. Alvarez-Muñiz, J. L. Feng, F. Halzen, T. Han and D. Hooper, hep-ph/0202081.

[9] J. Tanaka, T. Yamamura, S. Asai and J. Kanzaki, Study of black holes with the ATLAS detector at the LHC, ATL-PHYS-2003-037, November 2003.

[10] V. Cardoso, M. C. Espírito Santo, M. Paulos, M. Pimenta and B. Tomé, Astro. Phys. 22(2005)399.

[11] A. L. Read, CERN report 2000-005, 81 (2000).

[12] S. Giarrusso et al., Measurements of the UV nocturnal atmospheric background in the 300-400 nm wavelength band with the experiment BaBy during a transmediterranean balloon fight, Proc. of the $28^{\text {th }}$ ICRC, vol. HE, 849 (2003).

[13] K. Hagiwara, D. Zeppenfeld and S. Komamiya, Zeit. Phys. C29 (1985) 115; F. Boudjema, A. Djouadi and J.L. Kneur, Zeit. Phys. C57 (1993) 425. A. Djouadi, Zeit. Phys. C63 (1994) 317.

[14] M. C. Espirito Santo, M. Paulos, M. Pimenta, J. C. Romão and B. Tome, arXiv:hep-ph/0412345.

[15] W. Buchmuller, R. Ruckl and D. Wyler, Phys. Lett. B191, 442 (1987).

[16] E. Waxman and J. N. Bahcall, Phys. Rev. D 59, 023002 (1999) [hep-ph/9807282]; E. Waxman and J. N. Bahcall, hep-ph/9902383; K. Mannheim et al., astro-ph/9812398.

[17] W. Adam et al., DELPHI 2004-024 CONF 699, Contributed paper to ICHEP2004, Beijing, August 2004. 